\title{
His bundle rhythms with retrograde conduction to the atria
}

\author{
S. Gavrilescu and C. Luca \\ From the First Medical Clinic, Institute of Medicine, Timişoara, Rumania
}

His bundle electrograms were repeatedly recorded in two patients with complex arrhythmias. In the first case the pacemaker was located in the lower part of the left bundle-branch, retrogradely activating the His bundle. Atrial standstill was associated with this mechanism. After four days, His bundle tachycardia occurred, with constant retrograde activation of the atria, and anterograde block. In the second patient, who had progressive degeneration of the conducting system, His bundle rhythm activated the ventricles with a 2:I block. There was synchronization between the pacemaker located in the His bundle, and the atria. As the disease progressed, dissociation between atrial, His bundle, and ventricular rhythms developed.

Concealed His bundle automatic activity may explain some complex arrhythmias which would remain unsolved if the His bundle potentials were not recorded.

The interpretation of atrioventricular nodal rhythms may be considerably aided by the use of His bundle electrograms. As a result some traditional concepts assuming the formation of automatic impulses in the AV junctional area have had to be modified. Damato and Lau (1969) have provided evidence that in some so-called nodal rhythms, the His bundle and not the AV node is the pacemaker site. In some upper nodal rhythms, Touboul, Clément, and Delahaye (197I) were able to show an $\mathrm{H}$ potential preceding a retrograde $p$ wave, and a prolonged $\mathrm{HV}$ interval.

In this report the value of His bundle electrograms in the analysis of complex arrhythmias arising in the His bundle and the fascicular system is presented.

\section{Methods}

The technique of His bundle recordings used in our laboratory has been reported in previous communications (Pop et al., 1972; Gavrilescu et al., 1973). His bundle potentials were recorded employing a tripolar electrode catheter inserted percutaneously through the femoral vein. Pacing at the site of electrical activity was used to validate His bundle potentials. Recordings were made on a 6-channel direct-writing electrocardiograph (NEK-2, Zwönitz, East-Germany), at a paper speed of roo $\mathrm{mm} / \mathrm{sec}$.

Received 28 November 1973.

\section{Case reports}

\section{Case I}

A 65-year-old woman had mitral valvular disease and chronic congestive heart failure. She was known to be in atrial fibrillation and was treated with digitalis.

On admission, the electrocardiogram revealed nodal rhythm (rate 5I a minute) and no detectable atrial activity (Fig. I). The QRS complexes were $0.08 \mathrm{sec}$ in duration and showed right axis deviation $\left(+120^{\circ}\right)$.

His bundle electrograms were recorded on two separate occasions. The first recording (Fig. 2) showed atrial standstill and a regular ventricular activity with a cycle length of II $50 \mathrm{msec}$. These ventricular complexes were followed by $\mathrm{H}$ potentials which appeared at $90 \mathrm{msec}$ from the beginning of the ventricular depolarization. At each third or fourth beat, a different type of ventricular activity, preceded by an $\mathrm{H}$ deflection and an $\mathrm{HV}$ interval of $50 \mathrm{msec}$, occurred at a cycle length of 850-950 msec. There was no difference on the surface electrocardiogram in the QRS complexes, whether preceded or followed by the $\mathrm{H}$ potentials.

The serum electrolytes were normal and digitalis toxicity was suspected. The patient was treated with bed rest and spironolactone. After four days the heart rate was about 90 a minute, and the rhythm slightly irregular. Irregular atrial activity could be seen on the surface electrocardiogram (Fig. 3).

The intra-atrial electrogram revealed an atrial tachycardia (cycle length $500 \mathrm{msec}$ ), with no constant relation to ventricular complexes (Fig. 4A). The second His bundle recording (Fig. $4 \mathrm{~B}$ ) showed $\mathrm{H}$ potentials 


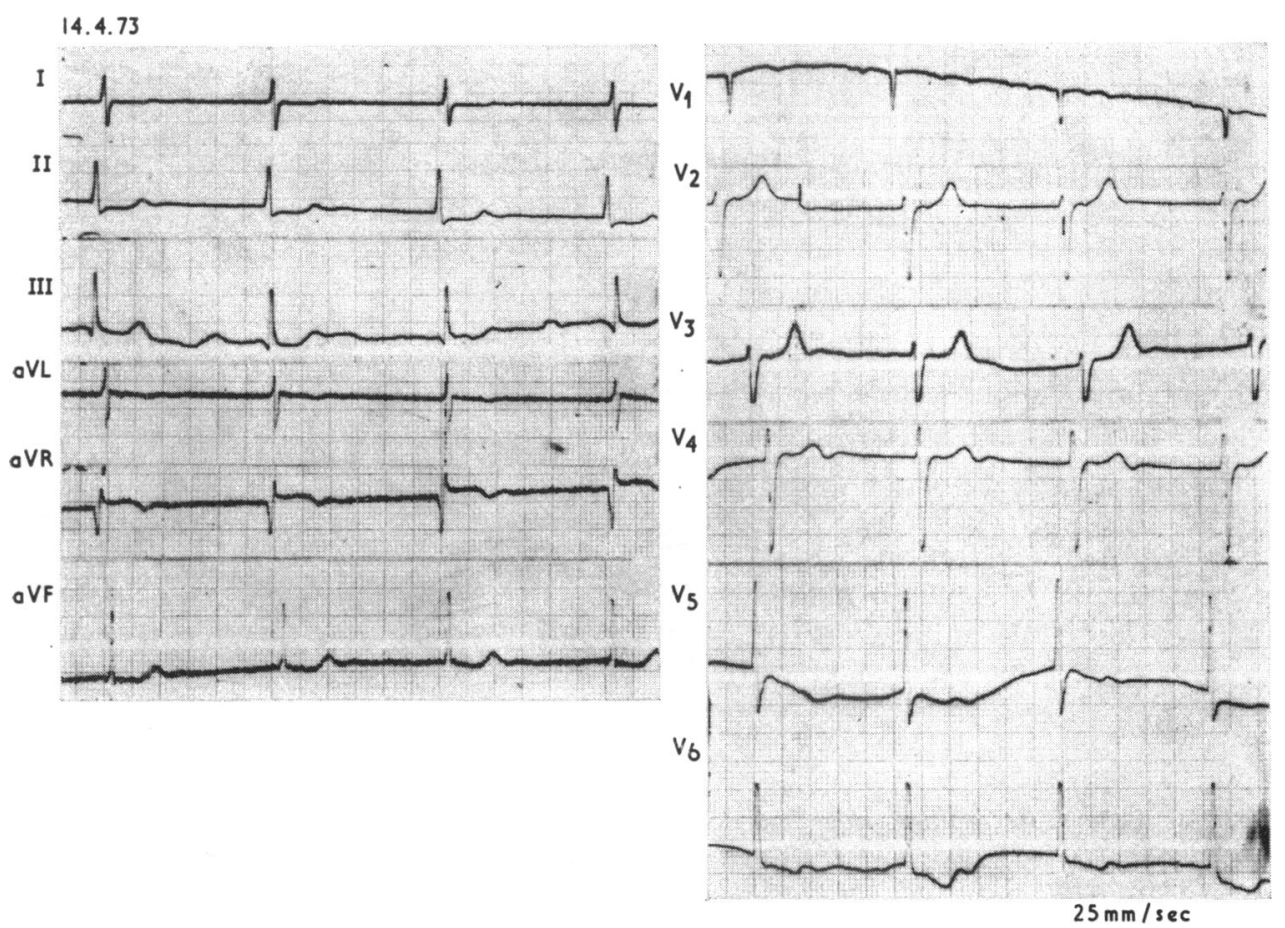

FIG. I Case I. The electrocardiogram on admission.

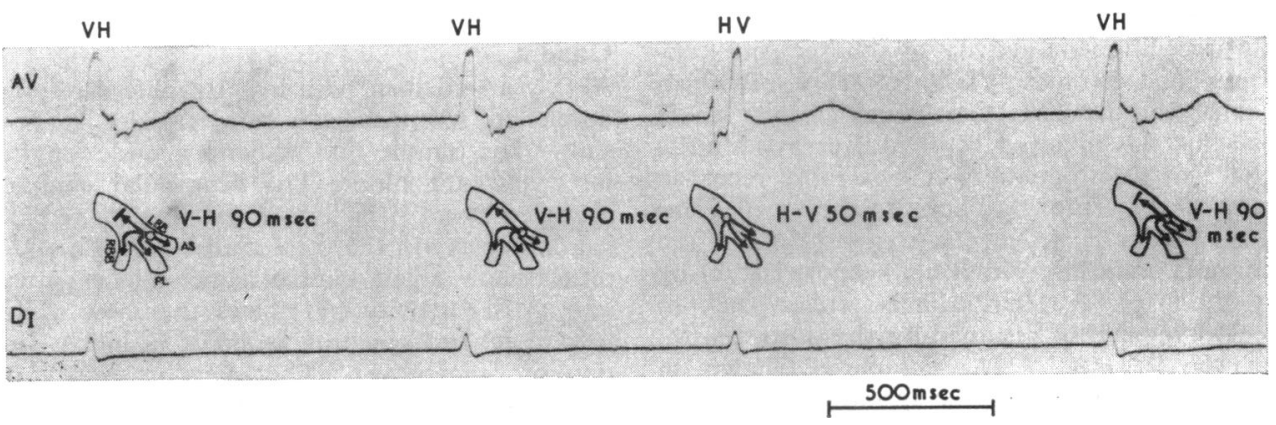

FIG. 2 Case 2: His bundle electrogram $(A V)$ and lead $I(D I):$ first recording. There is no atrial activity. VH are fascicular beats with retrograde activation of the His bundle. $H V$ is a His bundle beat. The diagrams show the proposed origin for each complex.

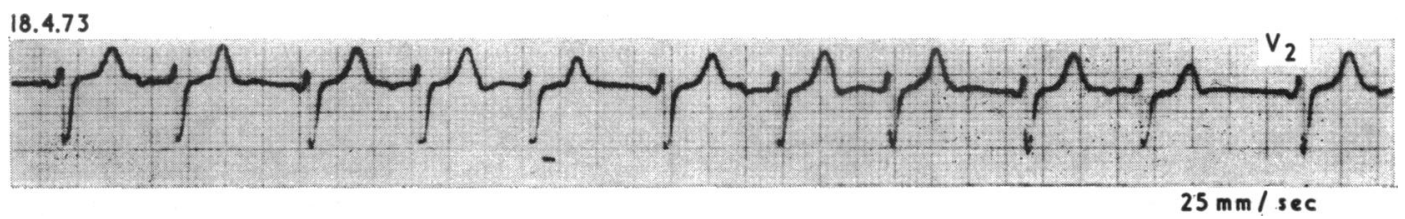

FIG. 3 Case 1 . Lead V2 showing the irregular atrial activity, four days after the recordings shown in Fig. $I$ and 2. 

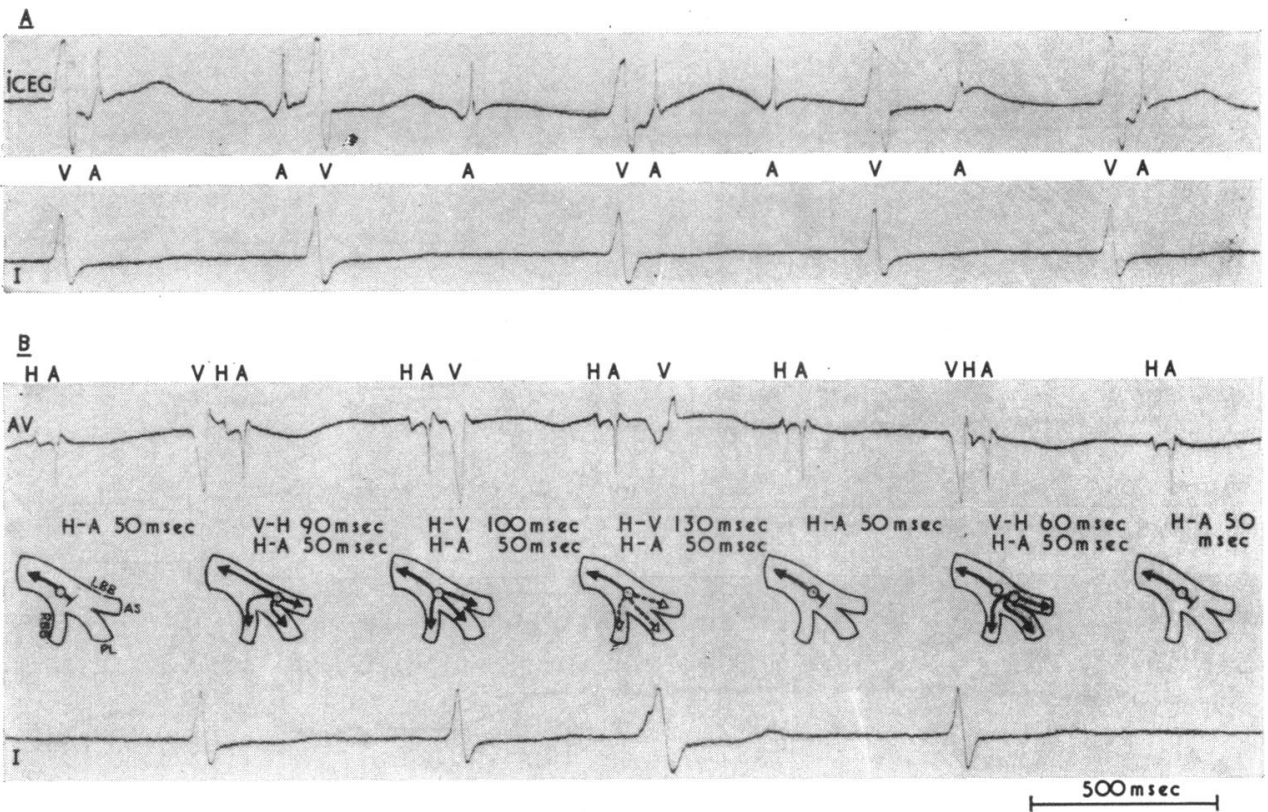

FIG. 4 Case I. Recordings taken four days after those shown in Fig. I and 2. A) Intra-atrial electrogram (ICEG) and lead I. B) His bundle electrogram (AV) and lead I. His bundle tachycardia with retrograde activation of the atria $(H A)$, and anterograde conduction defect is present. VHA are fascicular beats, the sixth complex is a fusion beat. The third, fourth, and fifth complexes ( $H A V, H A, V, H A$ ) show orthograde block of the Wenckebach type (see text for details). The diagrams show the proposed origin for each complex.

constantly preceding atrial activity by $50 \mathrm{msec}$. An exception is the second complex (VHA) from Fig. 4B which shows ventricular depolarization $90 \mathrm{msec}$ before an $\mathrm{H}$ deflection; this was followed $50 \mathrm{msec}$ later by an atrial activation. This electrogram resembles those recorded four days previously (Fig. 2). The third and fourth complexes (HAV) show $\mathrm{H}$ deflections $50 \mathrm{msec}$ before atrial activation, and preceding ventricular depolarization by 100 and $130 \mathrm{msec}$. A His-Purkinje-system block of Wenckebach type can be assumed for the third, fourth, and fifth (HAV, HAV, HA) complexes shown in Fig. 4B. This is supported by the appearance of a wide QRS complex on the surface electrocardiogram associated with the prolongation of the HV interval from 100 to $130 \mathrm{msec}$, demonstrating the slowing of anterograde conduction along the bundle-branches. The sixth complex (VHA) is a fusion beat between fascicular and His bundle rhythm.

In this patient, who probably suffered from digitalis toxicity and who had atrial standstill, the pacemaker was initially located in the lower part of the left bundlebranch, activating the His bundle retrogradely at a slow rate. His bundle premature beats were also seen. The second recording showed His bundle tachycardia with retrograde activation of the atria and anterograde block of Wenckebach type. Escape beats from the left divisional pacemaker were also observed.

\section{Case 2}

A 32-year-old man with hypertrophic cardiomyopathy developed spontaneous atrioventricular block. Fig. 5 shows His bundle rhythm with a cycle length of 900 msec and 2:I block. The ventricular complexes are preceded by an $\mathrm{H}$ deflection and a prolonged $\mathrm{HV}$ interval (140 msec) in the conducted beats. The QRS complexes show a left bundle-branch block pattern (O.13 sec). Atrial activity (A) shows the same cycle length (900 $\mathrm{msec}$ ) and synchronization of $\mathrm{H}$ and $\mathrm{A}$ depolarization by 'accrochage.' The injection of $0.25 \mathrm{mg}$ orciprenaline, a beta-adrenergic stimulating drug, shortened the cycle length of the His bundle rhythm to $60 \mathrm{msec}$. The anterograde block was unchanged, but retrograde activation of the atria occurred $60 \mathrm{msec}$ after each $\mathrm{H}$ deflection (Fig. 6). The retrograde conduction to the atria is demonstrated by the negative $\mathbf{P}^{\prime}$ waves in lead II and III, in contrast with the positive $P$ waves seen in Fig. 5.

A further shortening of the cycle length of the His bundle rhythm $(520 \mathrm{msec})$ under the effect of orciprenaline was associated with an increase of anterograde block to 3 : $\mathbf{I}$, while $\mathbf{H}$ depolarizations and atrial activity were incompletely dissociated (Fig. 7). There were isolated $\mathrm{H}$ potentials, blocked anterogradely and retrogradely, as well as retrogradely-conducted $\mathrm{H}$ deflections (HA) and $\mathrm{His}$ bundle echoes $\left(\mathrm{HVH}^{\prime}\right)$. Isolated capture beats (AHV) were also recorded. 


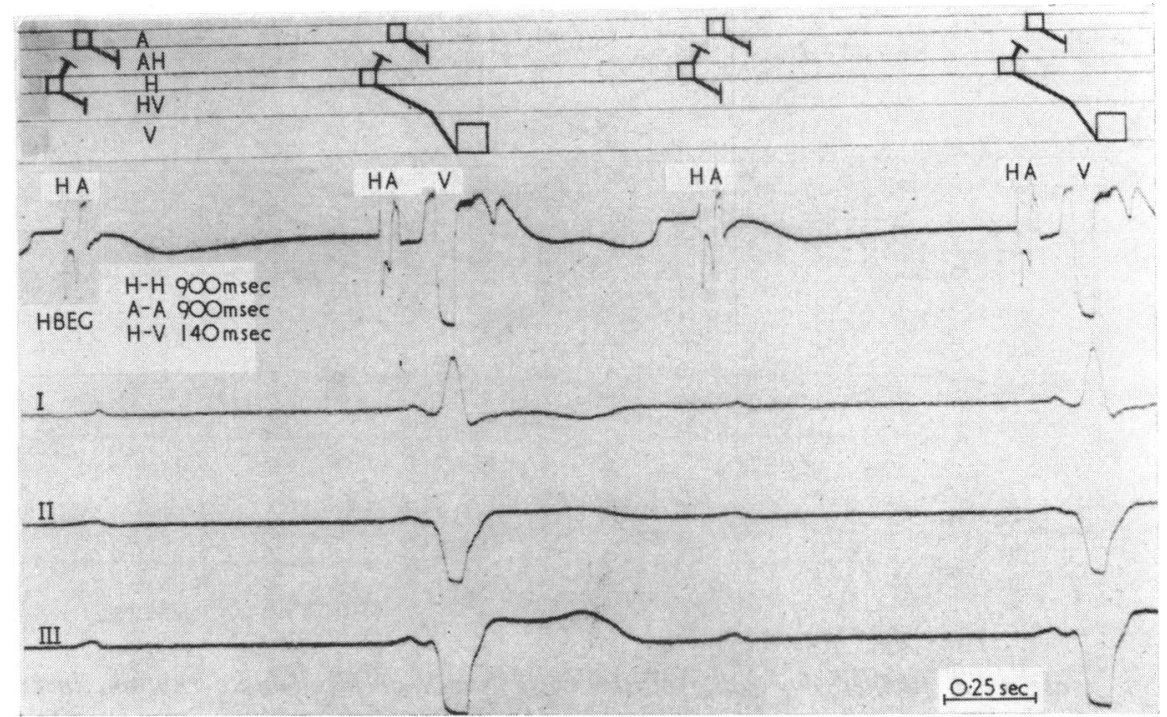

FIG. 5 Case 2. His bundle electrogram (HBEG) and leads I, II, and III. The His bundle rhythm shows a 2:I block and an HV interval of 140 msec in the conducted beats. The $H$ deflections are followed at 50 msec by atrial activity $(A)$. 'Accrochage' is the proposed mechanism for explaining synchronization (see text for details). The $P$ wave is positive in leads $I, I I$, and III. The relations between atrial, His bundle, and ventricular activity are represented on the upper diagrams.

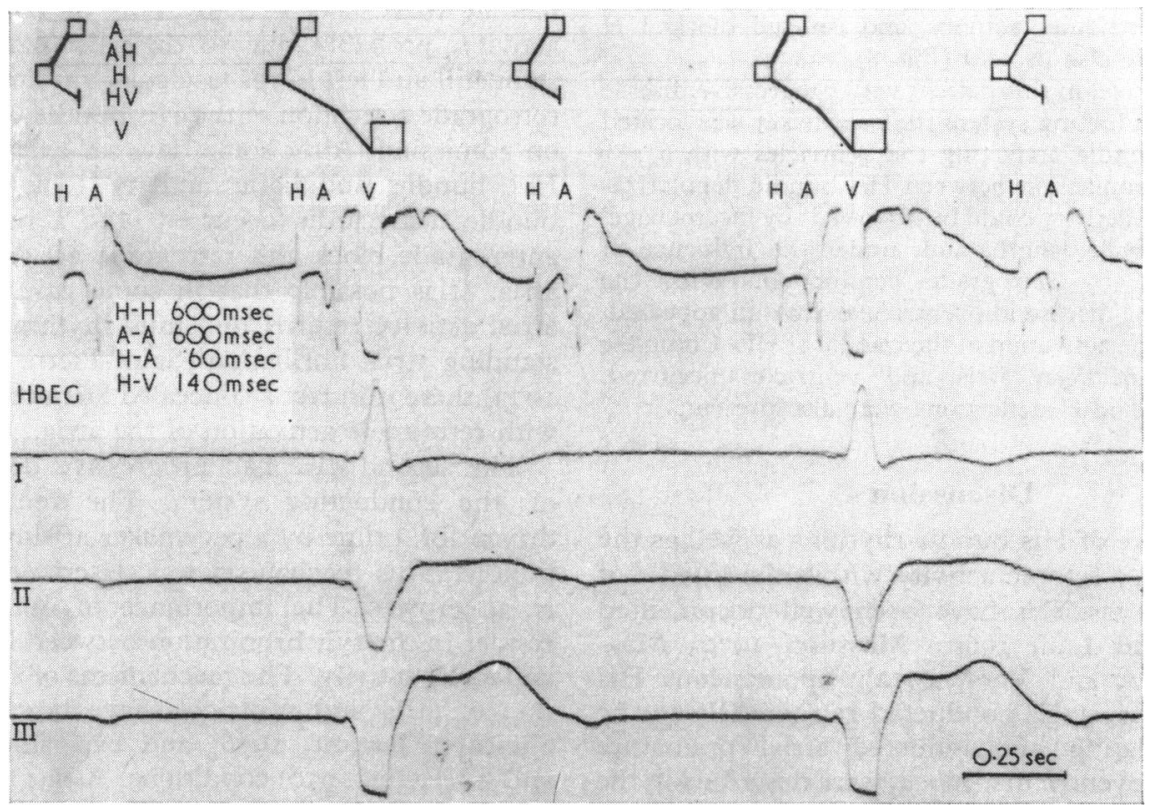

FIG. 6 Case 2. His bundle recording after the injection of orciprenaline. The cycle length of His bundle rhythm shortens from $900 \mathrm{msec}$ (Fig. 5) to $600 \mathrm{msec}$. The $H$ deflections are followed at $60 \mathrm{msec}$, by retrograde $P^{\prime}$ waves, which are negative in the peripheral leads. 


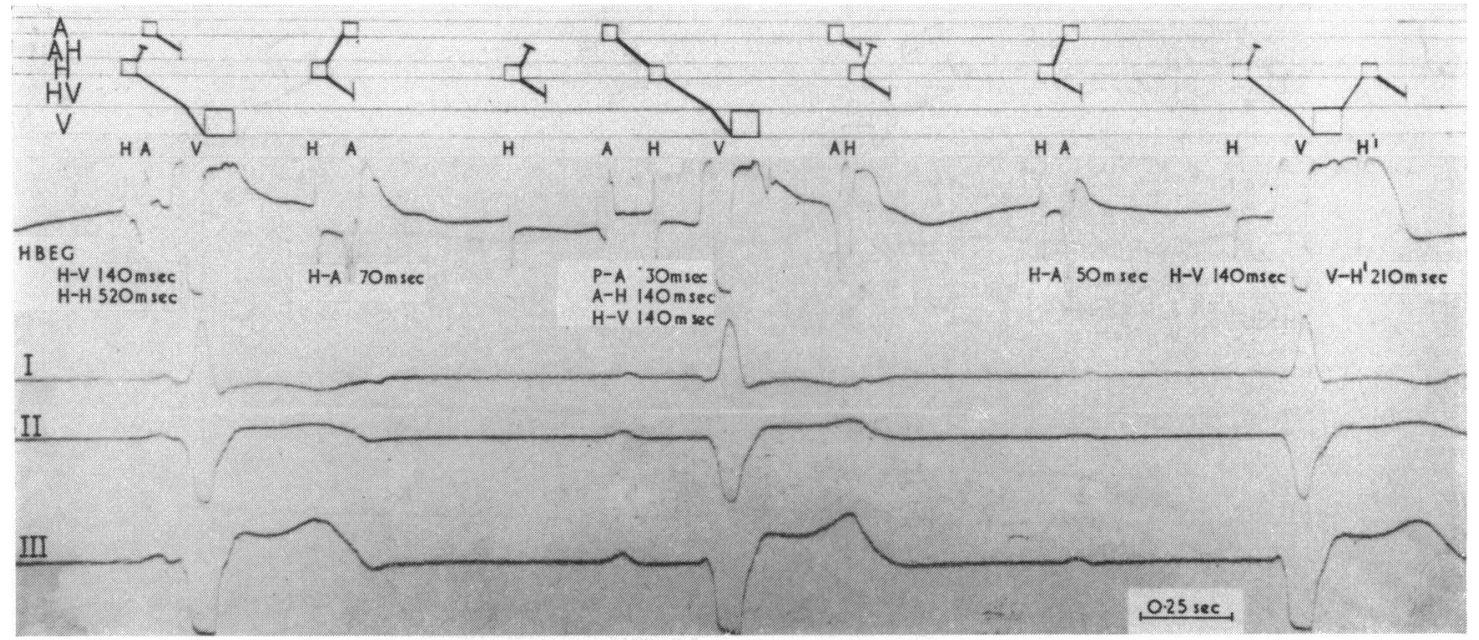

FIG. 7 Case 2. A further shortening of the cycle length of His bundle rhythm, increases anterograde block to 3:I. The relations between His bundle activity, atrial, and ventricular beats are shown on the upper diagram. There are anterograde conducted, retrograde conducted, and blocked $H$ potentials. AHV is an isolated capture beat. HVH' is a His bundle echo.

Three months later a second recording showed complete heart block with idioventricular rhythm (cycle length: I4IO msec, QRS duration: 0.14 sec) with retrograde activation of the $\mathrm{His}$ bundle. The $\mathrm{VH}^{\prime}$ interval was Ioo msec. There was complete dissociation between atrial and ventricular activity, and isolated blocked $\mathbf{H}$ potentials were also present (Fig. 8).

To summarize, in this patient with progressive disease of the AV conducting system the pacemaker was located in the His bundle activating the ventricles with a $2: I$ block. Synchronization between His bundle depolarization and atrial activity could be observed : by 'accrochage' at a longer cycle length, and, under the influence of orciprenaline, by retrograde conduction. With the progress of the disease idioventricular rhythm appeared, with retrograde activation of the bundle of His. Complete dissociation between atria and ventricles occurred. Isolated, blocked $\mathrm{H}$ deflections were also present.

\section{Discussion}

The existence of His bundle rhythms as well as the localization of ectopic activity within the bundle of His and its fascicles have been well documented (Damato and Lau, 1969; Massumi 1970; Massumi, Ertem, and Vera, 1972). Spontaneous His bundle extrasystoles conducted retrogradely to the atria, simulating nonconducted atrial premature beats or atrioventricular block, were described in the dog (Damato, Lau, and Bobb, I97I) and in man (Cannom et al., 1972; Eugster et al., 1973). The presence of narrow or wide QRS complexes during such rhythms can be explained by the site of impulse formation, aberrant conduction, and the speed of conduction in the fascicular system.

In the cases now reported, His bundle rhythm occurred under different circumstances. In the first patient there was a depression of the automatic activity, probably due to digitalis toxicity. Atrial standstill and left lower fascicular rhythm with slow retrograde activation of the His bundle were present on admission. After some days an enhancement of His bundle automatic activity took place. His bundle tachycardia occurred (I20 a minute) with anterograde block and retrograde activation of the atria. It is possible that in some cases where the atrial activity returns to 'sinus rhythm' after long standing atrial fibrillation (Saint-Pierre and Perrin, 1973) there is in fact a concealed His bundle rhythm with retrograde activation of the atria.

The second case had progressive determination of the conducting system. The ventricles were driven for a time by a pacemaker arising in the His bundle. This mechanism was described by Narula et al. (1970). The importance of our recordings resides in the synchronization between $\mathrm{H}$ potentials and atrial activity. The mechanisms of synchronization of atria and ventricles have been studied in clinical (Marriott, 1956) and experimental (Levy and Edelstein, 1970) conditions. As far as we know, this has not yet been demonstrated between His bundle and atrial activity.

The mechanism of synchronization between His bundle and atrial depolarizations was related to 


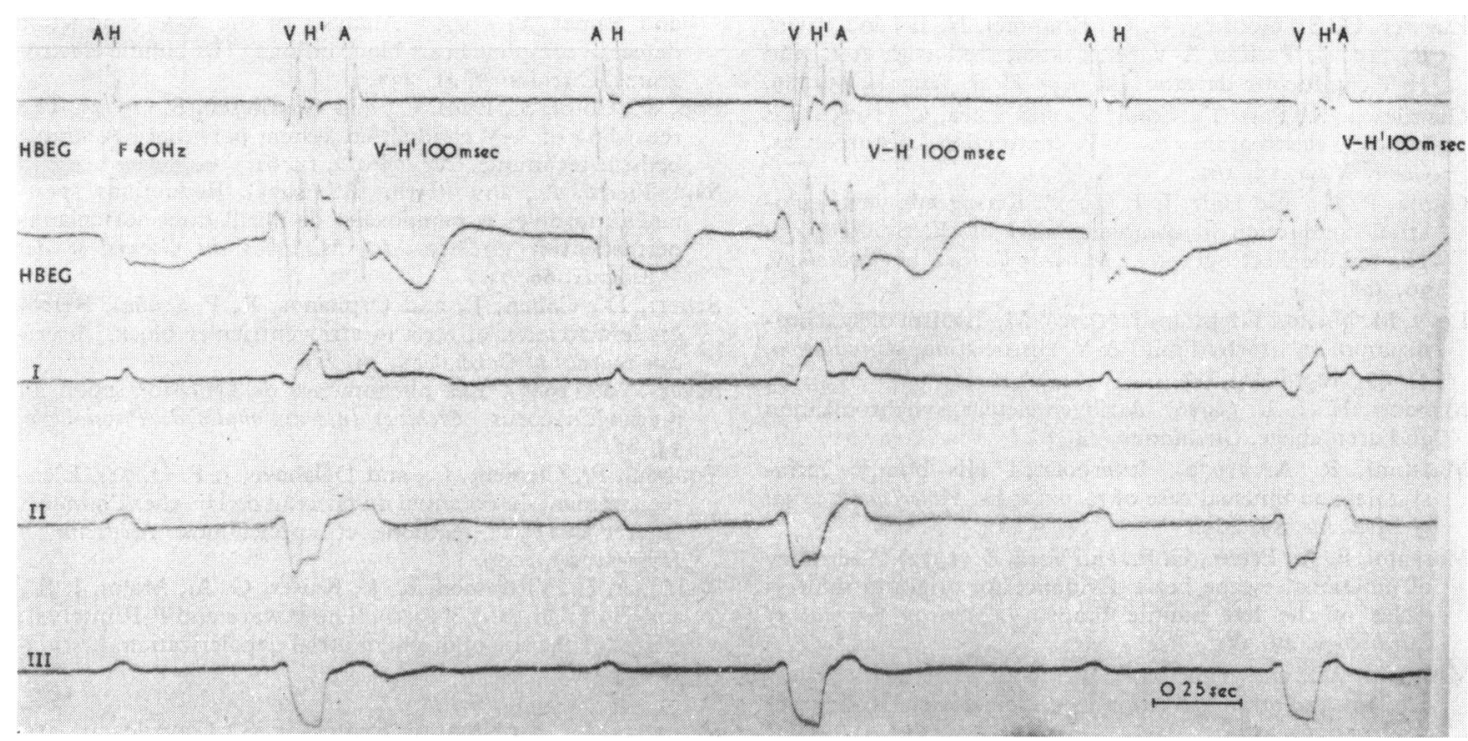

FIG. 8 Case 2. His bundle electrogram (HBEG) and leads I, II, III, second recording. Idioventricular rhythm with retrograde activation of the His bundle $\left(V H^{\prime}\right)$ is the dominant mechanism. A: atrial activity, $H$ : His bundle depolarizations (see text for details).

the cycle length of the His bundle rhythm. At a longer cycle length 'accrochage' was assumed to be the mechanism, while at shorter cycle lengths, under the influence of orciprenaline, retrograde activation of the atria took place.

On the basis of work with amphibian hearts Segers (1946) called 'accrochage' the process of synchronization which occurred when independent fragments of myocardium were placed in close contact. An electrical influence exerted by one tissue on the other may be responsible for this phenomenon. It is different from the capture of the heart chamber by retrograde conduction.

An alternative explanation for the two types of $P$ waves and HP relations found in our patient can be the activation of different ventriculoatrial pathways (Waldo et al., 1970).

The retrograde conduction from the ventricles to the atria is an infrequent event during atrioventricular block (Scherf, Cohen, and Orphanos, I964). Gupta and Haft (1972) studied ventriculoatrial conduction in a patient with complete heart block, using His bundle electrography. It was concluded that retrograde conduction could occur if the block was beyond the bundle of His and the AV node was intact. The retrograde pathway was supposed to be by muscular conduction. It was also suggested that retrograde $P^{\prime}$ waves were not the result of an impulse reaching the atria through the ventricular specialized system, but were a conse- quence of mechanical or electrotonic effects of ventricular contraction on a latent atrial focus (Mirowski and Tabatznik, 1970). However, our findings suggest that the ventricular impulses pass through the His bundle during its pathway towards the atria (Castillo and Castellanos, 197I).

The effect of orciprenaline, a drug that enhances the atrioventricular conduction, on the synchronization of His bundle and atrial activity is also of interest. Under its influence 'accrochage' was replaced by retrograde conduction. It is possible that the drug influenced the anterograde and retrograde pathways in different positions.

Concealed His bundle activity may explain some complex dysrhythmias which would remain unsolved if His bundle electrograms were not performed.

\section{References}

Cannom, D. S., Gallagher, J. J., Goldreyer, B. N., and Damato, A. N. (1972). Concealed bundle of His extrasystoles simulating nonconducted atrial premature beats. American Heart fournal, 83, 777.

Castillo, C., and Castellanos, A. (1971). Retrograde activation of the His bundle in the human heart. American fournal of Cardiology, 27, 264 .

Damato, A. N., and Lau, S. H. (1969). His bundle rhythm. Circulation, 40, 527.

Damato, A. N., Lau, S. H., and Bobb, G. (197I). Cardiac arrhythmias simulated by concealed bundle of His extrasystoles in the dog. Circulation Research, 28, 316. 
Eugster, G. S., Godfrey, C. C., Brammel, H. L., and Pryor, R. (1973). Pseudo A-V block associated with A-H and H-V conduction defects. American Heart fournal, 85, 789.

Gavrilescu, S., Pop, T., Cotoi, S., and Luca, C. (1973). His bundle electrogram in $\mathrm{A}-\mathrm{V}$ conduction disturbances. Cor et Vasa, 15, 161.

Gupta, P. K., and Haft, J. I. (1972). Retrograde ventriculoatrial conduction in complete heart block. Studies with His bundle electrography. American fournal of Cardiology, 30, 408.

Levy, M. N., and Edelstein, J. (1970). Mechanism of synchronization in isorhythmic A-V dissociation. Circulation, 4I-42, Suppl. III, 99.

Marriott, H. J. L. (1956). Atrioventricular synchronization and accrochage. Circulation, 14, 38.

Massumi, R. A. (1970). Interpolated His bundle extrasystoles: an unusual case of tachycardia. American fournal of Medicine, 49, 265.

Massumi, R. A., Ertem, G. E., and Vera, Z. (1972). Aberrancy of junctional escape beats. Evidence for origin in the fascicles of the left bundle branch. American fournal of Cardiology, 29, 35 ז.

Mirowski, M., and Tabatznik, B. (1970). The spatial characteristics of atrial activation in ventriculoatrial excitation. Chest, 57, 9.

Narula, S. O., Scherlag, B. J., Javier, R. P., Hildner, F. J., and Samet, P. (1970). Analysis of the A-V conduction defect in complete heart block utilizing His bundle electrograms. Circulation, 41, 437.

Pop, T., Cotoi, S., Luca, C., and Gavrilescu, S. (1972). The recording of A-V conduction system potentials. A simple bedside technique. Cor et Vasa, 14, 61.

Saint-Pierre, A., and Perrin, A. (1973). Réductions spontanées, tardives et paradoxales de fibrillations auriculaires permanentes. Archives des Maladies du Coeurs et des Vaisseaux, 66, 7I.

Scherf, D., Cohen, J., and Orphanos, R. P. (1964). Retrograde activation of atria in atrioventricular block. American fournal of Cardiology, 13, 219.

Segers, M. (1946). Les phénomènes de synchronisation au niveau du coeur. Archives Internationales de Physiologie, $54,87$.

Touboul, P., Clément, C., and Delahaye, J. P. (I97I). L'enregistrement du potential du faisceau de His chez l'homme. Interêt de cette méthode et applications. Médecine et Hygiène, 29, 1003 .

Waldo, A. L., Vitikainen, K. J., Kaiser, G. A., Malm, J. R., and Hoffman, B. F. (1970). The P wave and P-R interval; effects of the site of origin of atrial depolarization. Circulation, 42, 653 .

Requests for reprints to Professor S. Gavrilescu, str. Feuerbach Io, Timişoara, Rumania. 